\title{
Phi meson in dense matter
}

\author{
C. M. Ko, P. Lévai, ${ }^{*}$ and X. J. Qiu ${ }^{\dagger}$ \\ Cyclotron Institute and Physics Department, Texas A\&M University, College Station, Texas 77843 \\ C. T. Li \\ Physics Department, National Taiwan University, Taipei, Taiwan 10764, China
}

(Received 3 September 1991)

\begin{abstract}
The effect of the kaon loop correction to the property of a phi meson in dense matter is studied in the vector dominance model. Using the density-dependent kaon effective mass determined from the linear chiral perturbation theory, we find that with increasing baryon density the phi meson mass is reduced slightly while its width is broadened drastically.
\end{abstract}

PACS number(s): 21.30. $+\mathrm{y}, 21.90 .+\mathrm{f}$

In relativistic heavy-ion collisions, the nuclear matter can be compressed to densities which are many times that in normal nuclei. This has recently generated great interests in theoretical studies of hadron properties under extreme conditions [1]. In studies with both Nambu-Jona-Lasinio model [2] and QCD sum rules $[1,3]$, it has been found that hadron masses in general decrease with increasing density as a result of the partial restoration of chiral symmetry. The masses of pseudoscalar Goldstone bosons such as pion and kaon, however, do not depend much on the density $[2,4]$. But, in linear chiral perturbation theory, it has been shown that the effective mass of the kaon (also antikaon) in dense matter decreases with increasing nuclear density $[5,6]$. This is because nucleons act on kaon as an effective scalar field as a result of the explicit chiral symmetry breaking. The decreasing kaon mass in dense matter affects its production rate in heavy-ion collisions. Indeed, it has been shown [7] that this would lead to an enhanced production of kaons and may be responsible for the large $K^{+} / \pi^{+}$ratio observed in the Brookhaven National Laboratory experiments [8].

As the phi meson decays mainly into a kaon and antikaon pair, its property in dense matter is expected to be modified as well. Lissauer and Shuryak [9] have studied the change of the phi meson width in a hot matter of zero baryon density and have found that it increases by a factor of 2 due to the interaction of the kaon with pions. In their study, they have assumed that both kaon and phi masses do not change in the medium. Bi and Rafelski [10] have also found an increase of the phi meson width in dense matter by taking into account the in-medium kaon mass of Refs. [5,6] but keeping the phi meson mass unchanged. In this paper, we shall study both the mass and width of the phi meson in dense matter using the vector dominance model [11] and taking into account the

\footnotetext{
${ }^{*}$ On leave from KFKI, Budapest, Hungary.

†Permanent address: Institute of Nuclear Research, Academia Sinica, Shanghai 201800, China.
}

density-dependent kaon effective mass as given by the linear chiral perturbation theory $[5,6]$.

According to Refs. [5,6], the kaon effective mass in the medium can be expressed as

$$
m_{K}^{*}=m_{K}\left(1-\frac{\rho_{B}}{\rho_{c}}\right)^{1 / 2}
$$

with

$$
\rho_{c}=f_{K}^{2} m_{K}^{2} / \Sigma^{K N},
$$

where $m_{K}$ is the kaon mass in free space and $f_{K} \sim 93$ $\mathrm{MeV}$ is the kaon decay constant. The $K N$ sigma term $\Sigma^{K N}$ is defined by

$$
\Sigma^{K N}=\frac{1}{2}\left(m_{u}+m_{s}\right)\langle N|\bar{u} u+\bar{s} s| N\rangle
$$

with $m_{u}$ and $m_{s}$ being, respectively, the up and strange quark masses. There is considerable uncertainty in the strangeness content of the nucleon [12], but the lower and upper limits on $\rho_{c}$ can be obtained by taking $\langle N|\bar{s} s| N\rangle=\langle N|\bar{u} u| N\rangle \sim 3.75$ and by taking $\langle N|\overline{s S}| N\rangle=0$. The result is

$$
2.5 \rho_{0}<\rho_{c}<5 \rho_{0} .
$$

In the following, we shall choose $\rho_{c} \approx 5 \rho_{0}$, corresponding to zero strangeness content for the nucleon.

There is also a vector potential for a kaon in the nuclear matter [13]. Its strength is $\frac{1}{3}$ of the nucleon meanfield vector potential. Since this potential is repulsive for kaon and attractive for antikaon, it has thus no effect on phi meson which decays into a kaon and antikaon pair.

The interaction of the phi meson with kaon can be
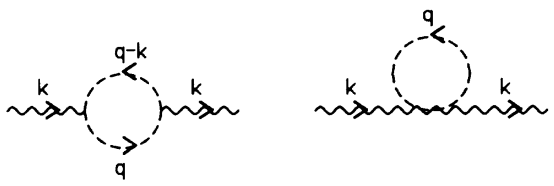

FIG. 1. The phi meson self-energy diagrams. The wavy and dashed lines denote, respectively, the phi meson and the kaon. 
studied in the vector dominance model [11]. The Lagrangian is given by

$$
L=\frac{1}{2}\left|D_{u} K\right|^{2}-\frac{1}{2} m_{K}^{2}|K|^{2}-\frac{1}{4} \phi_{\mu \nu} \phi^{\mu \nu}+\frac{1}{2} m_{\phi}^{2} \phi_{\mu} \phi^{\nu},
$$

where $K$ is the kaon field, $\phi_{\mu \nu}=\partial_{\mu} \phi_{\nu}-\partial_{\nu} \phi_{\mu}$ is the phi meson field strength, and $D_{\mu}=\partial_{\mu}-i g_{\phi} \phi_{\mu}$ is the covariant derivative. The phi meson mass is denoted by $m_{\phi}$. To include the medium effect on the kaon mass, we simply replace $m_{K}$ in the above equation by $m_{K}^{*}$.

The one-loop self-energy of the phi meson is shown by the diagrams in Fig. 1 and is given by

$$
\Pi^{\mu v}(k)=i g_{\phi}^{2} \int \frac{d^{4} q}{(2 \pi)^{4}}\left(\frac{(2 q-k)^{\mu}(2 q-k)^{v}}{\left(q^{2}-m_{K}^{* 2}+i \epsilon\right)\left[(q-k)^{2}-m_{K}^{* 2}+i \epsilon\right]}-\frac{2 g^{\mu v}}{q^{2}-m_{K}^{* 2}+i \epsilon}\right) .
$$

This integral is divergent but can be evaluated using dimensional regularization [14]. The result is

$$
\begin{aligned}
\Pi^{\mu v}(k)=\frac{g_{\phi}^{2}}{48 \pi^{2}}\left(k^{\mu} k^{v}-k^{2} g^{\mu v}\right) & {\left[\left(1-\frac{4 m_{K}^{* 2}}{k^{2}}\right]^{3 / 2}|\ln | \frac{\sqrt{1-4 m_{K}^{* 2} / k^{2}}+1}{\sqrt{1-4 m_{K}^{* 2} / k^{2}}-1} \mid-i \pi \theta\left(k^{2}-4 m_{K}^{* 2}\right)\right] } \\
& \left.+\frac{8 m_{K}^{* 2}}{k^{2}}+\ln \frac{m_{K}^{* 2}}{m_{k}^{2}}+C\right],
\end{aligned}
$$

where $C$ is the renormalization constant.

As in Ref. [14] for the rho meson, the phi meson self-energy can be written as

$$
\Pi^{\mu \nu}(k)=F(k) P_{L}^{\mu \nu}+G(k) P_{T}^{\mu \nu}
$$

in terms of the transverse $P_{T}^{00}=P_{T}^{0 i}=P_{T}^{i 0}=0, P_{T}^{i j}=\delta^{i j}-k^{i} k^{j} / \mathbf{k}^{2}$ and longitudinal $P_{L}^{\mu v}=k^{\mu} k^{v} / k^{2}-g^{\mu v}-P_{T}^{\mu \nu}$ projection tensors. Then one finds that

$F(k)=G(k)=\frac{g_{\phi}^{2}}{48 \pi^{2}} k^{2}\left(\left[1-\frac{4 m_{K}^{* 2}}{k^{2}}\right)^{3 / 2}\left(\ln \left|\frac{\sqrt{1-4 m_{K}^{* 2} / k^{2}}+1}{\sqrt{1-4 m_{K}^{* 2} / k^{2}}-1}\right|-i \pi \theta\left(k^{2}-4 m_{K}^{* 2}\right)\right)+\frac{8 m_{K}^{* 2}}{k^{2}}+\ln \frac{m_{K}^{* 2}}{m_{K}^{2}}+C\right)$,

and the phi meson propagator becomes

$$
D^{\mu \nu}\left(k^{2}\right)=-\frac{P_{L}^{\mu \nu}}{k^{2}-m_{\phi}^{2}-F}-\frac{P_{T}^{\mu \nu}}{k^{2}-m_{\phi}^{2}-G}-\frac{k^{\mu} k^{v}}{m_{\phi}^{2} k^{2}} .
$$

From Eq. (10), we find that the phi meson mass $m_{\phi}^{*}$ in the medium is determined by

$$
m_{\phi}^{* 2}-m_{\phi}^{2}-\operatorname{Re} F\left(m_{\phi}^{* 2}\right)=0,
$$

and its width $\Gamma_{\phi}^{*}$ is given by

$$
\Gamma_{\phi}^{*} \approx \frac{\operatorname{Im} F\left(k^{2}=m_{\phi}^{* 2}\right)}{m_{\phi}^{*}\left(1-\partial \operatorname{Re} F /\left.\partial k^{2}\right|_{k^{2}=m_{\phi}^{* 2}}\right)},
$$

where

$$
\frac{\partial \operatorname{Re} F}{\partial k^{2}}=\frac{g_{\phi}^{2}}{48 \pi^{2}}\left[\left(1-\frac{4 m_{K}^{* 2}}{k^{2}}\right]^{1 / 2}\left[1+\frac{2 m_{K}^{* 2}}{k^{2}}\right] \ln \left|\frac{\sqrt{1-4 m_{K}^{* 2} / k^{2}}+1}{\sqrt{1-4 m_{K}^{* 2} / k^{2}}-1}\right|+\ln \frac{m_{K}^{* 2}}{m_{k}^{2}}+1-\frac{4 m_{K}^{* 2}}{k^{2}}+C\right] .
$$

The factor $\left(1-\partial \operatorname{Re} F /\left.\partial k^{2}\right|_{k^{2}=m_{\phi}^{* 2}}\right)$ in the denominator of Eq. (12) takes into account the energy dependence of $\operatorname{Re} F$ as the width of a resonance is related to the distance of the pole from the real axis [15]. For vanishing kaon mass, i.e., $m_{K}^{*}=0, \operatorname{Re} F$ reduces to

$$
\operatorname{Re} F=\frac{g_{\phi}^{2}}{48 \pi^{2}} k^{2}\left(\ln \frac{k^{2}}{m_{K}^{2}}+C\right),
$$

and $\partial \operatorname{Re} F / \partial k^{2}=\operatorname{Re} F / k^{2}+g_{\phi}^{2} / 48 \pi^{2}$.
The renormalization constant $C$ is fixed by $\operatorname{Re} F\left(k^{2}=m_{\phi}^{2}\right)=0$ in the free space while the phi-kaon coupling constant $g_{\phi}$ is determined from the partial width of phi meson decaying into the $K \bar{K}$ pair. Using $m_{\phi}=1020 \mathrm{MeV}, \Gamma_{\phi}=4.41 \mathrm{MeV}$, and $m_{K}=495 \mathrm{MeV}$ in the free space, we obtain $C=-1.89$ and $g_{\phi}^{2} / 4 \pi=4.45$.

Taking $\rho_{c}=5 \rho_{0}$, we have calculated the phi meson mass and width in the matter and they are shown in Figs. 2 and 3. It is seen in Fig. 2 that the phi meson mass decreases slightly with increasing density except near the 


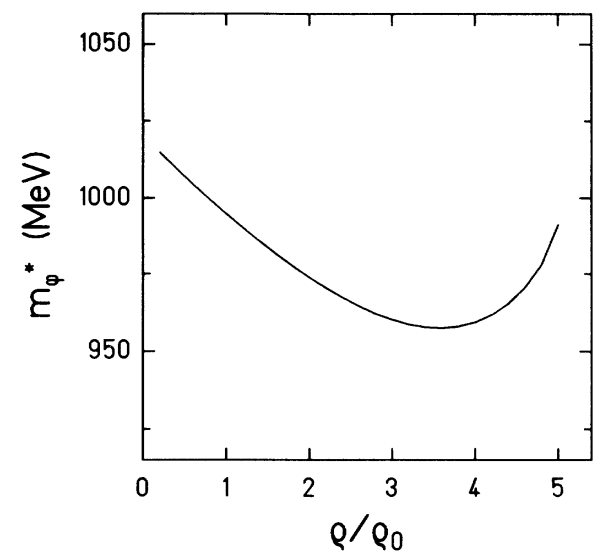

FIG. 2. The mass of phi meson as a function of matter density.

critical density $\rho_{c}$ where the phi meson mass increases again with the density but remains below the free mass. This change of the phi meson mass in dense matter is not included in Ref. [10]. The width shown in Fig. 3 increases with the density and becomes an order of magnitude larger than its value in the free space. The density effect on the phi meson width is much larger than the temperature effect studied in Ref. [9]. We note that the phi meson mass and width remain unchanged beyond the critical density. It has been shown in Ref. [16] that the decreasing phi meson mass in dense matter enhances its production in heavy-ion collisions and may account for the large $\phi / \omega$ ratio measured in the CERN experiments [17].

If we take the strangeness content of the nucleon to be nonzero, then the critical density at which the kaon mass vanishes will be reduced. This will make the minimum phi meson mass in Fig. 2 to occur at even lower density. For nonvanishing strangeness content of the nucleon, Hatsuda and Lee [3] have also found using the QCD sum rules method that the phi meson mass decreases with the density. A consistent calculation which includes both the

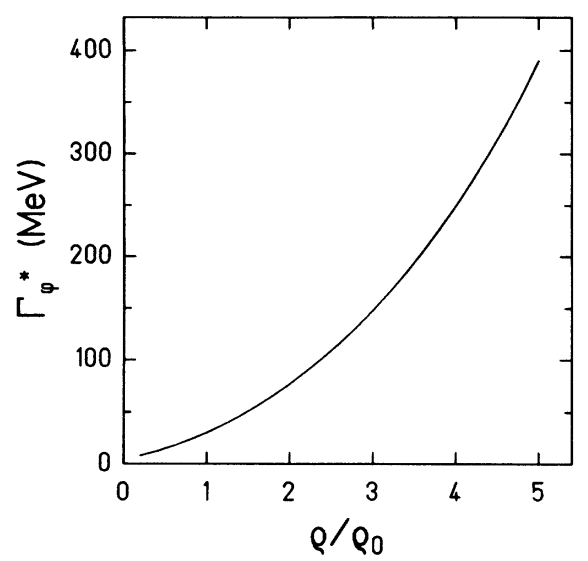

FIG. 3. The width of phi meson as a function of matter density.

QCD effect and the kaon loop correction discussed here would be very useful but is beyond the scope of the present study.

In summary, we have studied the kaon loop correction to the property of a phi meson in dense matter in the vector dominance model by including the change of the kaon mass in the medium as suggested by the chiral perturbation theory. We find that this leads to a decrease of the phi meson mass in dense matter. The phi meson width is seen to increase drastically with increasing baryon density when the kaon mass becomes small. Its value is already more than an order of magnitude larger than that in the free space at a moderate density of $2 \rho_{0}$. This change in the phi meson property in dense matter can be investigated experimentally via the dilepton invariant mass spectrum in high-energy heavy-ion collisions in which a dense matter is formed in the initial stage.

This work was supported in part by the National Science Foundation under Grant No. PHY-8907986 and the Welch Foundation under Grant No. A-1110.
[1] G. E. Brown, Nucl. Phys. A522, 397c (1991), and references therein.

[2] V. Bernard, U. G. Meissner, and I. Zahed, Phys. Rev. Lett. 59, 966 (1987); V. Meissner and U. G. Meissner, Phys. Lett. B 227, 465 (1989).

[3] T. Hatsuda and S. H. Lee (submitted to Phys. Rev. Lett.)

[4] T. Kunihiro, Nucl. Phys. B351, 593 (1991).

[5] D. B. Kaplan and A. E. Nelson, Phys. Lett. B 175, 57 (1986).

[6] G. E. Brown, K. Kubodera, and M. Rho, Phys. Lett. B 192, 273 (1987).

[7] C. M. Ko, Z. G. Wu, L. H. Xia, and G. E. Brown, Phys. Rev. Lett. 66, 2577 (1991); Phys. Rev. C 43, 1881 (1991).

[8] T. Abbott et al., Phys. Rev. Lett. 64, 847 (1990); 66, 1567 (1991).
[9] D. Lissauer and E. V. Shuryak, Phys. Lett. B 253, 15 (1991).

[10] P. Z. Bi and J. Rafelski, Phys. Lett. B 262, 485 (1991).

[11] J. J. Sakurai, Currents and Mesons (University of Chicago Press, Chicago, 1969).

[12] C. W. Wong, D. Vuong, and K. C. Chu, Nucl. Phys. A422, 635 (1990).

[13] G. E. Brown, C. M. Ko, and K. Kubodera, Z. Phys. A (in press).

[14] C. Gale and J. Kapusta, Phys. Rev. D 43, 3080 (1991).

[15] C. Mahaux, P. F. Bortignon, R. A. Broglia, and C. H. Dasso, Phys. Rep. 120, 48 (1985).

[16] C. M. Ko and B. H. Sa, Phys. Lett. B 258, 6 (1991).

[17] NA38 Collaborations, J. P. Guilland et al., Nucl. Phys. A525, 449c (1991). 